\title{
The Right Ventricle During the Acute Respiratory Distress Syndrome Revisited by Echocardiography
}

\author{
Vincent Caille and Antoine Vieillard-Baron*
}

\author{
Intensive Care Unit, University hospital Ambroise Paré, Assistance Publique des Hôpitaux de Paris, 9 avenue Charles \\ de Gaulle, 92104 Boulogne, France \\ Université de Versailles Saint Quentin en Yvelines, Faculté de Médecine Paris Ile de France Ouest, 78000 Saint \\ Quentin en Yvelines, France
}

\begin{abstract}
We illustrate the valuable information provided by echocardiography for hemodynamic monitoring and for optimizing ventilatory strategies, during ARDS. Although the transthoracic and the transesophageal routes can be used, we always prefer, in the absence of contraindications, to perform transesophageal echocardiography.

ARDS includes numerous affections which brutally damage the interface between the distal airway tract and pulmonary vascular bed. Two factors combine to produce right ventricular systolic overload, the pathologic features of the syndrome per se and mechanical ventilation.

Acute cor pulmonale (ACP) reflects the severity of the pulmonary disease, but may also be caused or exacerbated by an aggressive and unsuitable ventilatory strategy. With tidal volume limitation, the incidence of ACP has declined to $25 \%$. Providing that ventilatory management is adapted to right ventricular function, ACP is no longer significantly associated with increased mortality. If not, it is demonstrated that right ventricular dysfunction is actually associated with a poor prognosis.

In conclusion, whereas some have promoted a lung protective approach, echocardiography allows us to promote a right ventricular protective approach, by adapting respiratory settings to right ventricular function, which is key in the prognosis of these patients.
\end{abstract}

Keywords: ARDS, hemodynamics, right ventricle, echocardiography.

\section{INTRODUCTION}

In recent years, bedside use of Doppler echocardiography has supplanted invasive procedures in assessing cardiac function in critically ill patients. Pulmonary artery catheterization, an invasive procedure, gives indirect information related to right-sided heart function. This technique was gradually compromised, the measures being of debatable reliability in mechanical ventilation [1]. A qualitative and repetitive echocardiographic evaluation, by a simple visualization in real time of the kinetics and size of cardiac cavities, has been shown to be essential in assessing right-sided heart afterloading [2]. In addition, it should be considered that right ventricular dysfunction may affect left ventricular function, not only by limiting left ventricular preload, but also by adverse systolic and diastolic interactions via the intraventricular septum and the pericardium (ventricular interdependence). An experienced critical care intensivist with a sufficient echocardiographic background can immediately establish a functional diagnosis and aid clinical decision-making. In this clinical review we illustrate the valuable information provided by

*Address correspondence to this author at the Intensive Care Unit, University hospital Ambroise Paré, Assistance Publique des Hôpitaux de Paris, 9 avenue Charles de Gaulle, 92104 Boulogne, France; Tel: +33149095603; E-mail: antoine.vieillard-baron@apr.aphp.fr echocardiography for hemodynamic monitoring and for optimizing ventilatory strategies, during ARDS, a situation well known to be associated with pulmonary hypertension and right heart dysfunction.

\section{PHYSIOLOGY AND PATHOPHYSIOLOGY}

Systolic function of the right ventricle may be represented by the coupling between "intrinsic" contractility of the right ventricle and its afterload [3]. In physiologic conditions, contractility is somewhat secondary, because simple negative pleural pressure produced by breathing promotes blood flow through the pulmonary circulation and ensures sufficient pulmonary venous return. The right ventricle acts as a passive conduit. This is possible because the right ventricle ejects blood into a low-resistance, highcompliance circuit. The volume of blood present in the pulmonary circulation is the filling reserve of the left ventricle. A reduction in this volume immediately affects the left ventricular preload and may cause decreased left ventricular stroke volume and finally circulatory failure.

Conversely, in pathologic conditions in which there is some increase in pulmonary vascular resistance, "intrinsic" contractility becomes essential to maintain an optimal coupling between the right ventricle and the pulmonary circulation and then to promote pulmonary blood flow. Unfortunately, unlike the left ventricle, adaptation of the 
right ventricle is limited. It can be argued that right ventricular systolic function is sensitive: even a slight increase in pulmonary vascular resistance can overload a normal right ventricle, thereby impairing its systolic function. In response, because of a significantly low diastolic elastance, the right ventricle dilates acutely [4]. It can be argued that right ventricular diastolic function is tolerant.

ARDS includes numerous affections which brutally damage the interface between the distal airway tract and pulmonary vascular be d. Two factors combine to produce right ventricular systolic overload. The first regards the pathologic features of the syndrome per se, which is associated with distal occlusion of the pulmonary arterial bed [5] and with pulmonary vascular remodeling (muscularization of usually non-muscularized arteries) mediated by hypoxia and hypercapnia (Fig. 1). The second factor is mechanical ventilation, which increases right ventricular outflow impedance because of the elevated transpulmonary pressure due to lung compliance impairment $[6,7]$. Increasing tidal volume increases right ventricular afterload during tidal ventilation, whereas increasing PEEP induces increased afterload during both tidal ventilation and expiration (Fig. 2) [8]. When right ventricular afterload is progressively increased, the related prolonged right ventricular contraction is responsible for a reversal in the transseptal pressure gradient, especially at end systole $[9,10]$. This explains why paradoxical septal motion can be observed.

As a consequence, pulmonary hypertension is common in ARDS [11]. In certain situations, it can lead to a pattern of
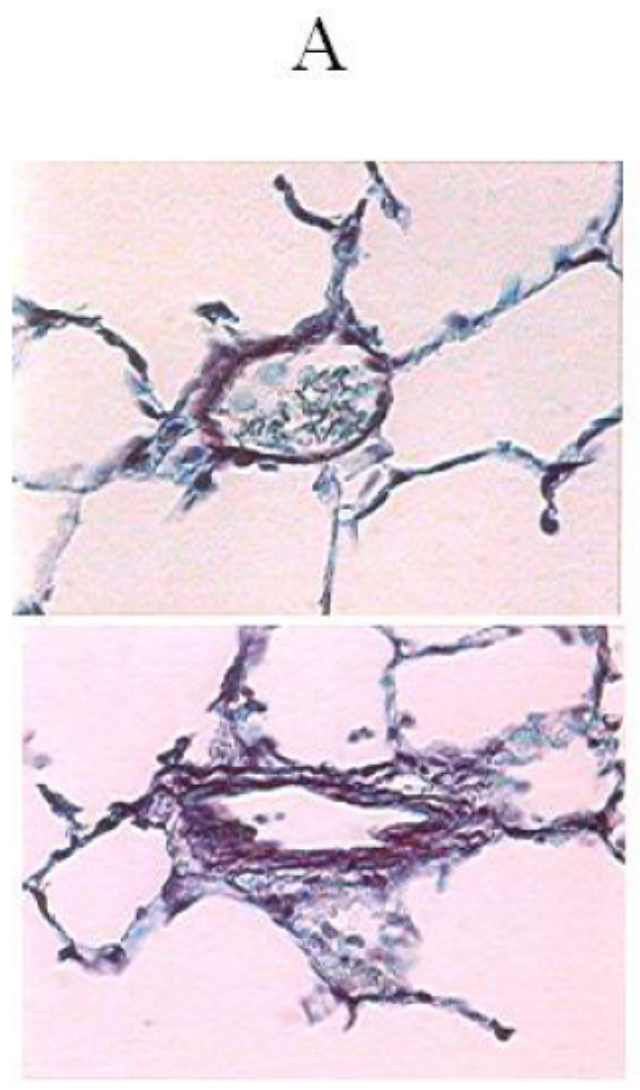

acute cor pulmonale (ACP), defined as the association between right ventricular enlargement, indicative of diastolic overload, and paradoxical septal motion, indicative of systolic overload (Fig. 3) [12]. The echocardiographic pattern of ACP complicating ARDS was first described in 1985 [13].

\section{ECHOCARDIOGRAPHIC EXAMINATION IN ARDS}

Although the transthoracic and the transesophageal routes can be used, we always prefer, in the absence of contraindications, to perform transesophageal echocardiography. In these mechanically ventilated patients, it is safe, minimally invasive and allows good reproducibility of the evaluation, whoever the operator, at different periods of time.

Echocardiographic examination of the right ventricle requires a long-axis view of the heart to evaluate the size of the cavity and a short-axis view to evaluate the septal kinetics. The examination can be completed by Doppler examination of the right ventricular ejection flow and of the backward flow across the tricuspid valve when present.

\section{Right Ventricular Size Assessment}

Right ventricular diastolic dimensions can be obtained by measuring right ventricular end-diastolic area in a long-axis view obtained by a transthoracic or transesophageal route (Fig. 3). The best way to quantify right ventricular dilatation is to measure the ratio between the right and left ventricular end-diastolic areas, which circumvents individual variation

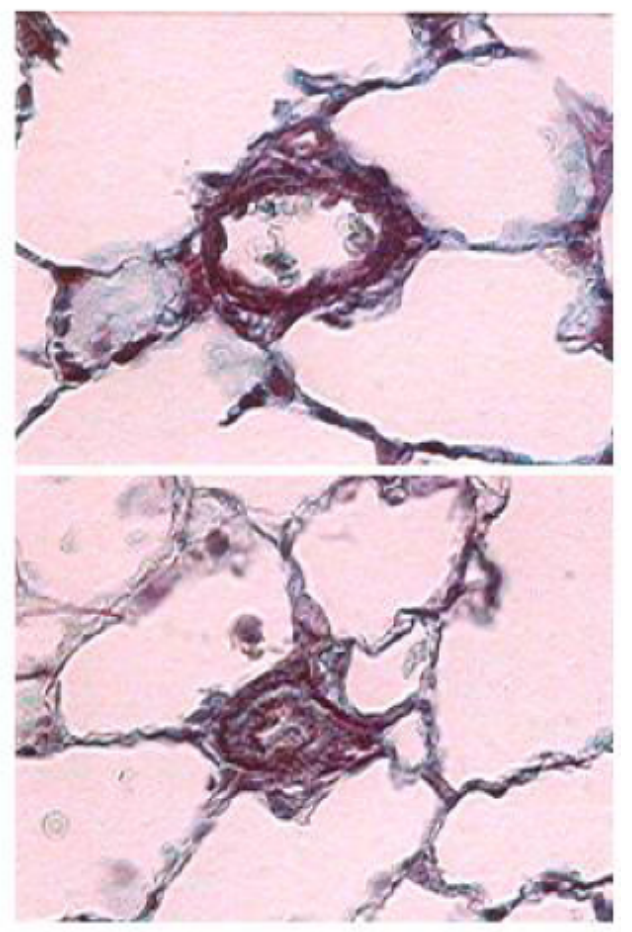

Fig. (1). Lung sections in a rat exposed to $21 \%$ oxygen (panel $\mathbf{A}$ ) and in a rat exposed to $10 \%$ oxygen for 10 days (panel $\mathbf{B}$ ). Whereas in rat A, pulmonary arteries were normally non-muscularized, pulmonary vascular remodeling occurred in rat B. 
A
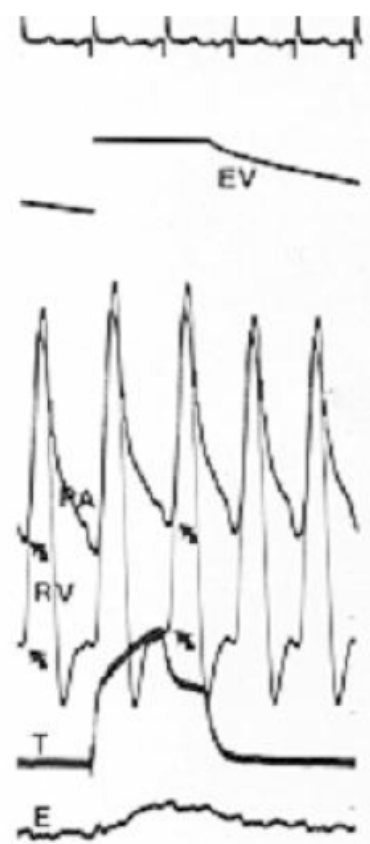

B

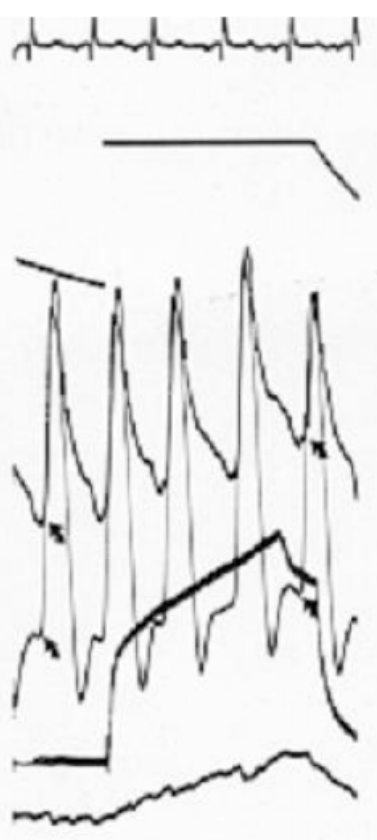

C
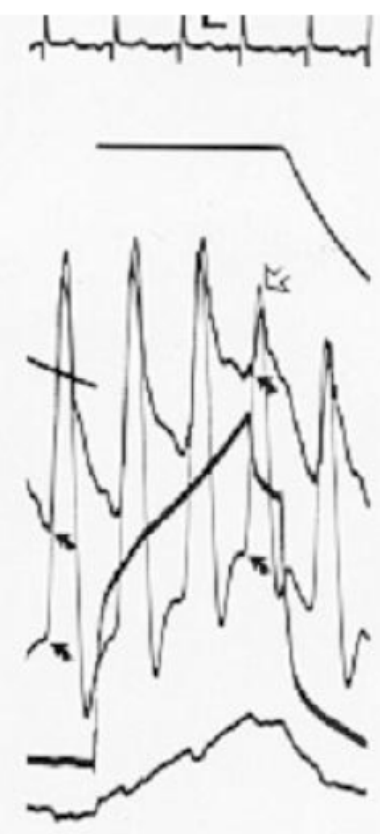

Fig. (2). Right heart catheterization in a patient mechanically ventilated for ARDS during an increase in tidal volume from a low level (panel A) to a high level (panel $\mathbf{C}$ ). Increasing tidal volume was responsible during insufflation for an increase in the isovolumetric contraction pressure (arrows) and for a decrease in pulmonary pulse. The two reflect a decrease in right ventricular stroke volume related to increased afterload. T: tracheal pressure, E: esophageal pressure, RV: right ventricular pressure, PA: pulmonary artery pressure.

in cardiac size. In a group of normal volunteers, we reported a mean normal ratio of $0.48 \pm 0.12$ [14]. We have thus defined moderate right ventricular dilatation as a ratio greater than 0.6 and major right ventricular dilatation as a ratio greater than or equal to 1 [12]. However, gradation of right ventricular dilatation by ventricular diastolic area ratio may be inoperative when chronic left ventricular dilatation is present, because of valvular disease or dilated cardiomyopathy.

\section{Septal Dyskinesia}

Paradoxical septal motion is best observed in a short-axis view recorded by either a parasternal or transgastric approach (Fig. 3). When present at end-systole, it reflects systolic overload of the right ventricle, as emphasized above. Most of the time, the evaluation of septal kinetics is qualitative, but the systolic eccentricity index has been proposed to "quantify" the systolic overload of the right ventricle [15]. A value close to 1 is normal, whereas a value higher than 1 reflects systolic overload of the right ventricle.

\section{Pulmonary Arterial Hypertension}

Pulmonary hypertension is usually associated with tricuspid regurgitation. Measurement of the maximal velocity of tricuspid backward flow with continuous wave Doppler allows estimation of systolic pulmonary artery pressure. A moderate elevation is usually observed, but the value should be viewed with caution because systolic pulmonary artery pressure also depends on right ventricular systolic fuction. In fact, in some extreme acute cor pulmonale, pulmonary hypertension may be lacking because major right ventricular failure with inefficient right ventricular contraction is responsible for markedly low cardiac output [16].

Interestingly, we have demonstrated in ARDS that the right ventricle is able in only a few days to thicken in response to an increase in pulmonary pressure. We described a moderate thickness of the right ventricular free wall after 2 days of mechanical ventilation [2].

Doppler examination of pulmonary artery flow velocity can reveal alterations of the flow due to acute pulmonary hypertension: a decrease in the velocity time integral, reflecting the decrease in right ventricular stroke volume, a reduction in acceleration time and sometimes a biphasic flow pattern (Fig. 4). Moreover, when pressure in the right atrium exceeds that in the left atrium, the foramen ovale may open. The detection of a patent foramen ovale requires contrast echocardiography and has been greatly improved by transesophageal echocardiography [17]. It can be also diagnosed using color-flow Doppler echocardiography.

\section{Left Ventricular Consequences of Acute Cor Pulmonale}

Sudden right ventricular enlargement in the stiff pericardial space causes left ventricular compression (Fig. 4). In consequence, the normal left ventricular end-diastolic volume is decreased in acute cor pulmonale [18]. Left ventricular filling impairment in acute cor pulmonale results from combined pulmonary vascular occlusion and septal displacement [19]. This acute preload deficit often produces or precipitates acute circulatory failure in this setting [18]. 


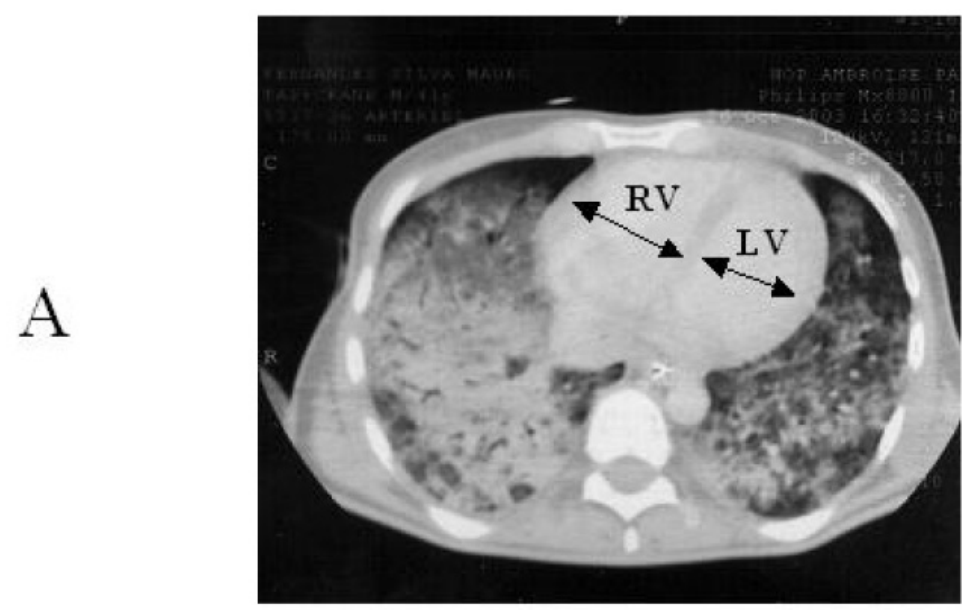

$\mathrm{B}$

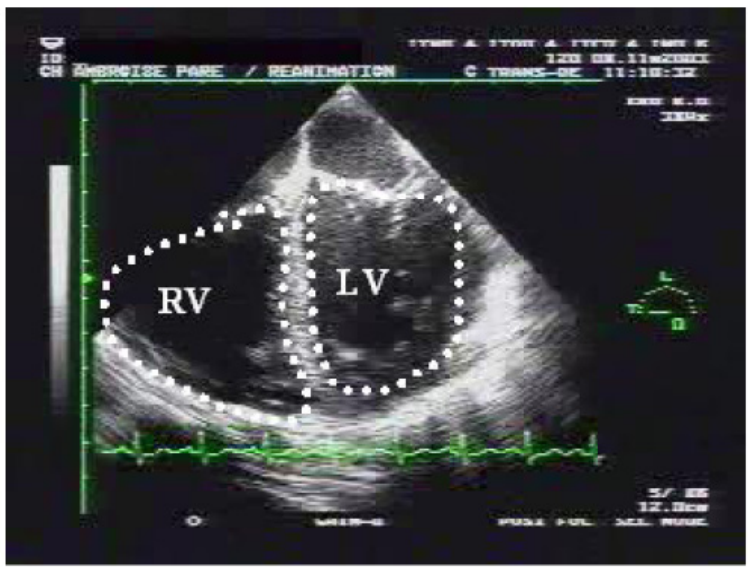

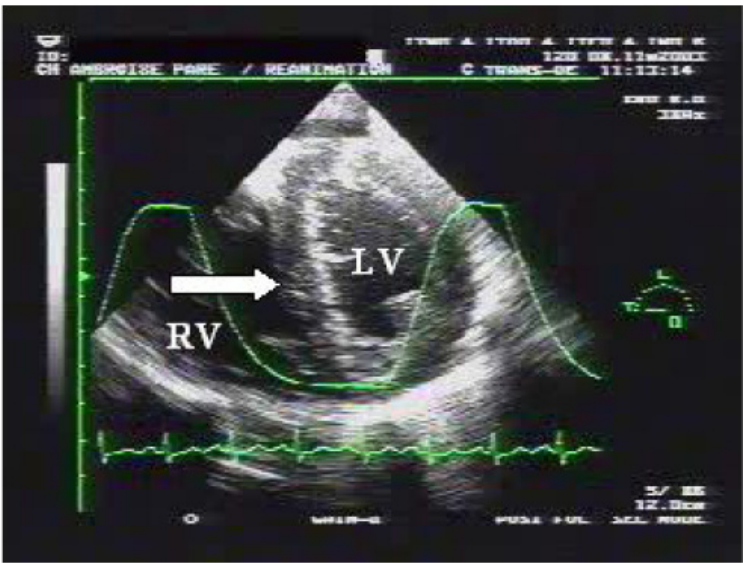

Fig. (3). Patient mechanically ventilated for severe ARDS. CT scan (panel A) showed severe bilateral lung injury, but also right ventricular dilatation. Transesophageal echocardiography demonstrated a pattern of acute cor pulmonale with dilatation of the right ventricle (panel B) and paradoxical septal motion in systole (panel $\mathbf{C}$, arrow). RV: right ventricle, LV: left ventricle.

The latter produces abnormal left ventricular relaxation, which is also evidenced by an abnormal mitral profile. The ratio of peak velocity during early diastolic filling ( $\mathrm{E}$ wave) to peak velocity of the atrial systole (A wave) is inverted in acute cor pulmonale complicating acute respiratory distress syndrome [18].

\section{WHY PROTECT THE RIGHT VENTRICLE IN PATIENTS WITH ARDS, AND HOW?}

When high tidal volumes were used $(13 \mathrm{~mL} / \mathrm{kg})$, the incidence of ACP was high (61\%) and this was associated with a poor prognosis [13]. In the most severe forms, with a right ventricle bigger than the left ventricle, we have even observed $100 \%$ mortality [13]. More recently, with tidal volume limitation, the incidence of ACP in acute respiratory distress syndrome has declined to $25 \%$ [18]. Providing that ventilatory management is adapted to right ventricular function, ACP is no longer significantly associated with increased mortality. If not, it is clearly demonstrated that right ventricular dysfunction is actually associated with a poor prognosis. We showed that mortality was not different in patients with or without ACP providing that the plateau pressure is maintained below $27 \mathrm{cmH}_{2} \mathrm{O}$, whereas there was a significant difference when plateau pressure was higher than $27 \mathrm{cmH}_{2} \mathrm{O}$ [20]. This was confirmed by Osman et al. who reported in a large series that right ventricular dysfunction was an independent parameter of mortality if ventilatory settings are not adapted [21]. So, many arguments underscore the need to adapt mechanical ventilation to right ventricular function. Observation of ACP may thus lead to suppression or limitation of the different factors known to favor right ventricular systolic overload, i.e. high plateau pressure, high PEEP and hypercapnia. Plateau pressure should be re-examined and maintained below $27 \mathrm{cmH}_{2} \mathrm{O}$, as explained above. PEEP should also be limited. The mean PEEP in our unit in ARDS is $7 \mathrm{cmH}_{2} \mathrm{O}$ 


\section{D2 $22 \mathrm{cmH}_{2} \mathrm{O}$

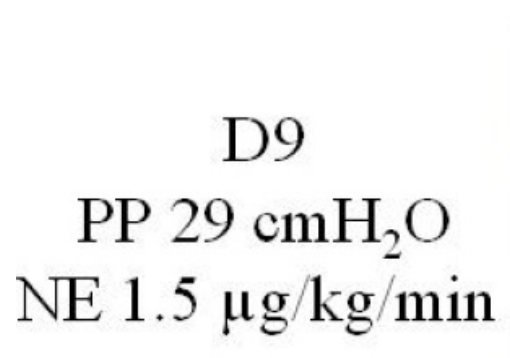

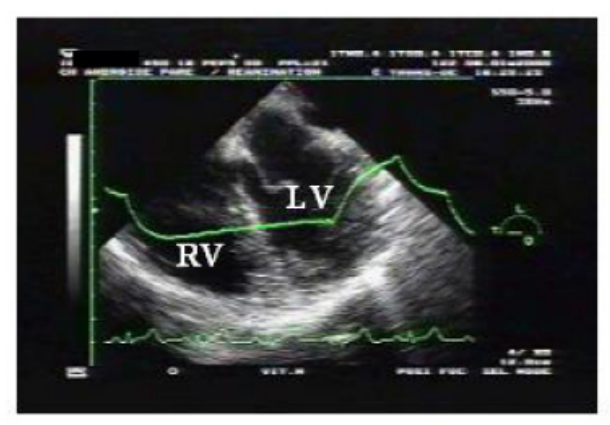
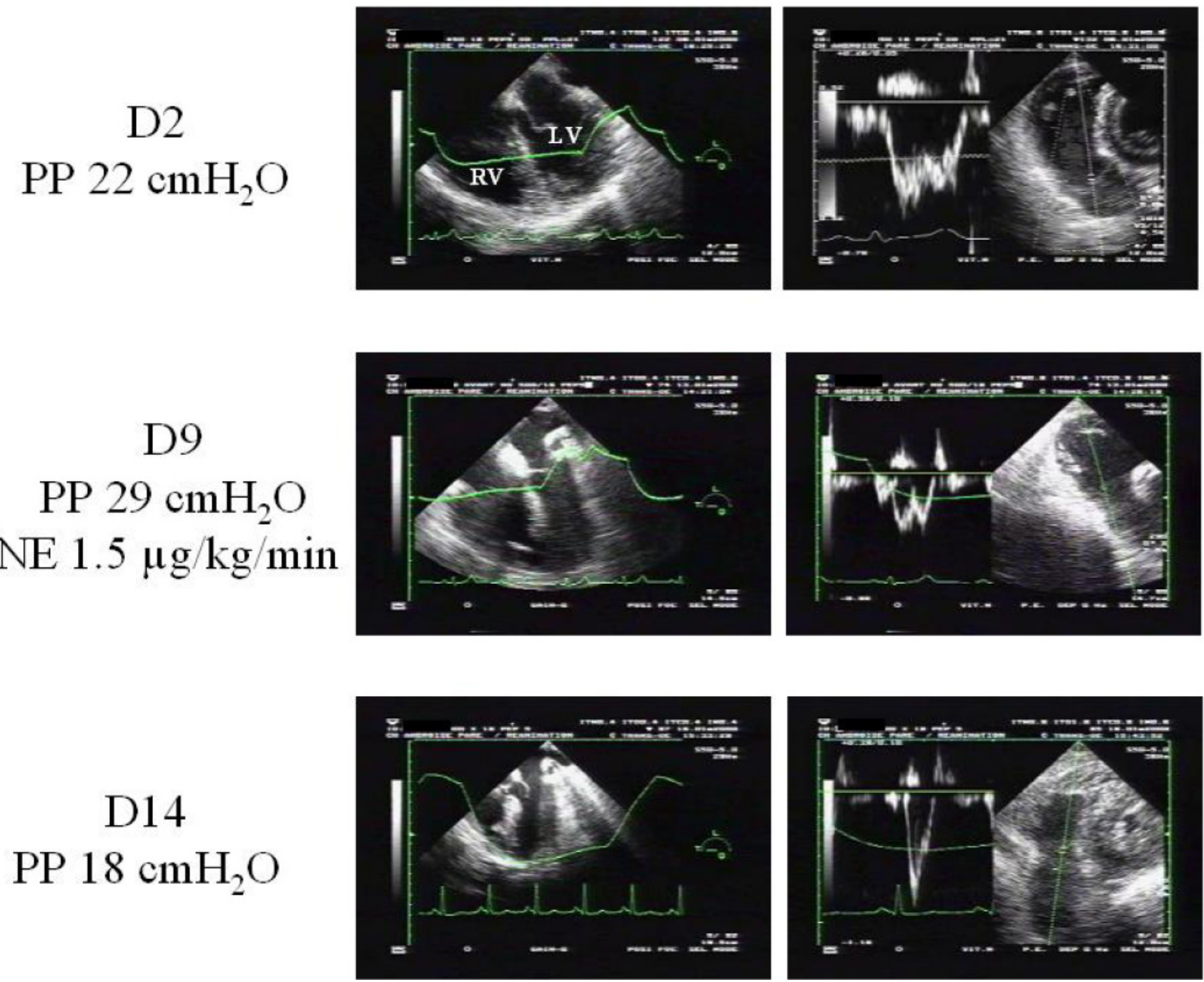

Fig. (4). Evolution of right ventricular function from admission (D2) to recovery (D14) in a patient ventilated because of severe ARDS related to pneumonia. At day 2, plateau pressure was $22 \mathrm{cmH}_{2} \mathrm{O}$. Hemodynamics were normal. Echocardiography demonstrated moderate dilatation of the right ventricle with an incipient biphasic pattern of the pulmonary flow. The right ventricular stroke volume was conserved. At day 9, following major deterioration in respiratory mechanics (plateau pressure $29 \mathrm{cmH}_{2} \mathrm{O}$ ), echocardiography demonstrated major dilatation of the right ventricle leading to strong restriction of the left ventricle. The pulmonary flow showed a clear biphasic pattern with a decrease in right ventricular stroke volume. The patient was in shock and required high-dose norepinephrine infusion. Finally, at day 14, after recovery of lung function, right ventricular function appeared normal and hemodynamics also. PP: plateau pressure, NE: norepinephrine, RV: right ventricle, LV: left ventricle.

because we always favor oxygen transport rather than oxygen content [22]. Intrinsic PEEP should absolutely be avoided. It is often caused by an excessive respiratory rate, an insidious cause of right ventricular afterloading [23]. Because $\mathrm{PaCO}_{2}$ is a well-known factor which promotes pulmonary hypertension, the presence of ACP may induce limitation of the hypercapnia. We recently reported very poor tolerance in terms of right ventricular function in ARDS patients subject to significant hypercapnia [24]. Hypercapnia must be limited first by increasing the respiratory rate, without inducing intrinsic PEEP, and second by removing the heat moisture exchanger and using instead a heated humidifier [25]. If PEEP reduction does not appear possible in the supine position without worsening hypoxemia, prone positioning should be considered on day 3. We demonstrated that this positioning strategy in the most severely ill patients, i.e. patients with a persistent $\mathrm{P} / \mathrm{F}$ ratio below $100 \mathrm{mmHg}$ after 2 days of mechanical ventilation, was responsible for a dramatic improvement in oxygenation and in respiratory mechanics [26]. Interestingly, we reported that it was also good for the right ventricle, by unloading it [27].

Finally, in a very few situations, after adapting respiratory settings to right ventricular function, NO inhalation can be tested in patients with persistent shock related to $\mathrm{ACP}$.

\section{CONCLUSION}

In conclusion, in ARDS, acute cor pulmonale reflects the severity of the pulmonary disease involving the microvasculature, but may also be caused or exacerbated by an aggressive and unsuitable ventilatory strategy. The classic clinical description of acute cor pulmonale has to be revised 
and completed by a more modern approach based on echocardiographic changes in the size and function of the heart. These echocardiographic findings allow us to assess the hemodynamic consequences and prognostic implications of acute respiratory distress syndrome. Whereas some have promoted a lung protective approach, especially based on high PEEP, echocardiography allows us to promote a right ventricular protective approach, by adapting respiratory settings to right ventricular function, which is key in the prognosis of these patients.

\section{REFERENCES}

[1] Jardin F, Bourdarias JP. Right heart catheterization at bedside: a critical view. Intensive Care Med 1995; 21: 291-5.

[2] Vieillard-Baron A, Prin S, Chergui K, Dubourg O, Jardin F. EchoDoppler demonstration of acute cor pulmonale at the bedside in the medical intensive care unit. Am J Respir Crit Care Med 2002; 166: 1310-9.

[3] Wauthy P, Pagnamenta A, Vassalli F, Naeije R, Brimioulle S. Right ventricular adaptation to pulmonary hypertension: an interspecies comparison. Am J Physiol Heart Circ Physiol 2004; 286: H1441-7.

[4] Laks MM, Garner D, Swan HJ. Volumes and compliances measured simultaneously in the right and left ventricles of the dog. Circ Res 1967; 20: 565-9.

[5] Zapol WM, Jones R. Vascular components of ARDS. Clinical pulmonary hemodynamics and morphology. Am Rev Respir Dis 1987; 136: 471-4

[6] Whittenberger JL, McGregor M, Berglund E, Borst HG. Influence of state of inflation of the lung on pulmonary vascular resistance. J Appl Physiol 1960; 15: 878-82.

[7] Vieillard-Baron A, Loubieres Y, Schmitt JM, Page B, Dubourg O, Jardin F. Cyclic changes in right ventricular output impedance during mechanical ventilation. J Appl Physiol 1999; 87: 1644-50.

[8] Jardin F, Brun-Ney D, Cazaux P, Dubourg O, Hardy A, Bourdarias JP. Relation between transpulmonary pressure and right ventricular isovolumetric pressure change during respiratory support. Cathet Cardiovasc Diagn 1989;16:215-20.

[9] Elzinga G, Piene H, de Jong JP. Left and right ventricular pump function and consequences of having two pumps in one heart. A study on the isolated cat heart. Circ Res 1980; 46: 564-74.

[10] Jardin F, Farcot JC, Boisante L, Curien N, Margairaz A, Bourdarias JP. Influence of positive end-expiratory pressure on left ventricular performance. N Engl J Med 1981; 304: 387-92.

[11] Zapol WM, Snider MT. Pulmonary hypertension in severe acute respiratory failure. N Engl J Med 1977; 296: 476-80.

[12] Jardin F, Dubourg O, Bourdarias JP. Echocardiographic pattern of acute cor pulmonale. Chest 1997; 111: 209-17.

[13] Jardin F, Gueret P, Dubourg O, Farcot JC, Margairaz A, Bourdarias JP. Two-dimensional echocardiographic evaluation of right ventricular size and contractility in acute respiratory failure. Crit Care Med 1985; 13: 952-6.
[14] Vieillard-Baron A, Page B, Augarde R, et al. Acute cor pulmonale in massive pulmonary embolism: incidence, echocardiographic pattern, clinical implications and recovery rate. Intensive Care Med 2001; 27: 1481-6.

[15] Ryan T, Petrovic O, Dillon JC, Feigenbaum H, Conley MJ, Armstrong WF. An echocardiographic index for separation of right ventricular volume and pressure overload. J Am Coll Cardiol 1985; 5: 918-27.

[16] Jardin F, Gurdjian F, Desfonds P, Fouilladieu JL, Margairaz A Hemodynamic factors influencing arterial hypoxemia in massive pulmonary embolism with circulatory failure. Circulation 1979; 59: 909-12.

[17] Konstadt SN, Louie EK, Black S, Rao TL, Scanlon P. Intraoperative detection of patent foramen ovale by transesophageal echocardiography. Anesthesiology 1991; 74: 2126.

[18] Vieillard-Baron A, Schmitt JM, Augarde R, et al. Acute cor pulmonale in acute respiratory distress syndrome submitted to protective ventilation: incidence, clinical implications, and prognosis. Crit Care Med 2001; 29: 1551-5.

[19] Jardin F, Dubourg O, Gueret P, Delorme G, Bourdarias JP. Quantitative two-dimensional echocardiography in massive pulmonary embolism: emphasis on ventricular interdependence and leftward septal displacement. J Am Coll Cardiol 1987; 10: 1201-6.

[20] Jardin F, Vieillard-Baron A. Is there a safe plateau pressure in ARDS? The right heart only knows. Intensive Care Med 2007;33: 444-7.

[21] Osman D, Monnet X, Castelain V, Anguel N, Warszawski J, Teboul JL, Richard C; French Pulmonary Artery Catheter Study Group. Incidence and prognostic value of right ventricular failure in acute respiratory distress syndrome. Intensive Care Med 2009; 35: 69-76.

[22] Schmitt JM, Vieillard-Baron A, Augarde R, Prin S, Page B, Jardin F. Positive end-expiratory pressure titration in acute respiratory distress syndrome patients: impact on right ventricular outflow impedance evaluated by pulmonary artery Doppler flow velocity measurements. Crit Care Med 2001; 29: 1154-8.

[23] Vieillard-Baron A, Prin S, Augarde R, et al. Increasing respiratory rate to improve $\mathrm{CO}_{2}$ clearance during mechanical ventilation is not a panacea in acute respiratory failure. Crit Care Med 2002; 30: 1407-12.

[24] Mekontso Dessap A, Charron C, Devaquet J, et al. Impact of acute hypercapnia and augmented positive end-expiratory pressure on right ventricle function in severe acute respiratory distress syndrome. Intensive Care Med 2009; 35: 1850-8.

[25] Prin S, Chergui K, Augarde R, Page B, Jardin F, Vieillard-Baron A. Ability and safety of a heated humidifier to control hypercapnic acidosis in severe ARDS. Intensive Care Med 2002; 28: 1756-60.

[26] Vieillard-Baron A, Rabiller A, Chergui K, et al. Prone position improves mechanics and alveolar ventilation in acute respiratory distress syndrome. Intensive Care Med 2005; 31: 220-6.

[27] Vieillard-Baron A, Charron C, Caille V, Belliard G, Page B, Jardin F. Prone positioning unloads the right ventricle in severe ARDS. Chest 2007; 132: 1440-6. 